Revista Brasileira de Agricultura Irrigada v.11, nº.7, p. 2076 - 2083, 2017

ISSN 1982-7679 (On-line)

Fortaleza, CE, INOVAGRI - http://www.inovagri.org.br

DOI: $10.7127 /$ rbai.v11n700710

Protocolo 710.17 - 28/06/2017 Aprovado em 17/10/2017

\title{
UTILIZAÇÃO DE SENSOR E TENSIÔMETRO NO MONITORAMENTO DA UMIDADE DO SOLO NA CULTURA DO FEIJOEIRO SOB DEFICIÊNCIA HÍDRICA
}

\author{
Edilson Ramos Gomes ${ }^{1}$, Renata Bruna dos Santos Coscolin ${ }^{2}$, Jéssica de Lima ${ }^{3}$, Enrique \\ Alonso Zuñiga ${ }^{4}$, Luz Maria Ruíz Machuca ${ }^{5}$, Fernando Broetto ${ }^{6}$
}

\section{RESUMO}

A água é insumo limitante à produção vegetal e exerce papel decisivo na produção de alimentos. As culturas agrícolas exigem valor mínimo de disponibilidade hídrica, que facultará às plantas o seu bom desenvolvimento e produção. Para manutenção do estado hídrico do solo faz-se necessário o uso de tecnologia de irrigação, como sensores ou equipamentos que podem reproduzir esta condição hídrica, determinante para a escolha do momento e quantidade de água a ser fornecida. Neste ensaio, utilizou-se duas qualidades de água e diferentes lâminas de irrigação, visando simular a deficiência hídrica moderada e severa na cultura do feijoeiro em estufa agrícola. Para monitorar o teor de água no solo e também para comparar métodos de medidas, utilizou-se tensiômetros instalados nos diferentes tratamentos propostos. Paralelamente, tomou-se medidas de teor volumétrico de água, a partir do sensor modelo smrty. Para verificar o efeito da deficiência hídrica, determinou-se parâmetros biométricos como altura de planta, diâmetro do caule, número de folhas e área foliar total a partir do florescimento do feijoeiro. Os métodos de análise do teor de água no solo foram equivalentes, justificando a utilização do sensor smrt-y, em substituição ao método tensiométrico. Os parâmetros biométricos analisados indicaram efeito de redução do crescimento do feijoeiro ocasionado pelo baixo teor de água no solo, notadamente ao final do ciclo da cultura.

Palavras-chave: manejo de irrigação, Phaseolus vulgaris L., sensor smrt-y

\footnotetext{
${ }^{1}$ Doutor em Agronomia (Irrigação e Drenagem), UNESP, Departamento de Engenharia Rural, Botucatu-SP, email: edilsonevj@hotmail.com

${ }^{2}$ Doutora em Agronomia (Irrigação e Drenagem), UNESP, Departamento de Engenharia Rural, Botucatu-SP, email: renata.coscolin@gmail.com

${ }^{3}$ Mestranda em Agronomia (Irrigação e Drenagem), UNESP, Departamento de Engenharia Rural, Botucatu-SP, e-mail: jessik1300@hotmail.com

${ }^{4}$ Doutorando em Agronomia (Irrigação e Drenagem), UNESP, Departamento de Engenharia Rural, Botucatu-SP, e-mail: ing_sierra-alta@hotmail.com

${ }^{5}$ Doutoranda em Agronomia (Irrigação e Drenagem), UNESP, Departamento de Engenharia Rural, Botucatu-SP, e-mail: luzmy_rm@hotmail.com

${ }^{6}$ Doutor em Ciências, Prof. da UNESP, Departamento de Química e Bioquímica, Botucatu-SP, e-mail: broetto@ibb.unesp.br
} 


\title{
USE OF SENSOR AND TENSIOMETER IN THE MONITORING OF SOIL MOISTURE IN COMMON BEAN UNDER WATER DEFICIT
}

\begin{abstract}
Water is an input limiting plant production and plays a decisive role in food production. Agricultural crops require a minimum amount of water availability, which will enable the plants to be well developed and productive. To maintain the water status of the soil, it is necessary to use irrigation technology, such as sensors or equipment that can reproduce this water condition, which is decisive for choosing the moment and quantity of water to be supplied. In this trial, two water qualities and different irrigation slides were used to simulate the moderate and severe water deficit in the common bean crop in an agricultural greenhouse. In order to monitor soil water content and also to compare measurement methods, tensiometers installed in different treatments were used. At the same time, measurements of water volumetric content were taken from the model sensor smrt-y. To verify the effect of water deficit, biometric parameters were determined as plant height, stem diameter, number of leaves and total leaf area from bean flowering. The methods of soil water content analysis were equivalent, justifying the use of the smrt-y sensor, instead of the tensiometric method. The biometric parameters analyzed showed reduction effect of bean growth caused by the low water content in the soil, especially at the end of the crop cycle.
\end{abstract}

Keywords: irrigation management, Phaseolus vulgaris L., smrt-y sensor

\section{INTRODUÇÃO}

Mundialmente, o Brasil está entre os grandes produtores e consumidores de grãos de feijão. No mercado interno apresenta alta visibilidade, pois o feijão retrata uma importante fonte de proteína e ferro na alimentação da população, além da valor socioeconômico (AZEVEDO et al. 2015). Assim, o uso racional dos recursos hídricos na irrigação do feijoeiro com a finalidade de maximizar a sua produção é de suma importância.

Neste contexto, o uso dos recursos hídricos na agricultura irrigada é cada vez mais preocupante, considerando-se que as previsões de disponibilidade de água indicam crescente escassez e baixa qualidade deste recurso. Assim, torna-se necessário o uso de técnicas e equipamentos de irrigação com elevados níveis de eficiência. Para atender a esses critérios, as preocupações com os equipamentos de irrigação e monitoramento da água no solo são indispensáveis tanto no processo de planejamento quanto no de implantação dos sistemas. Portanto é fundamental o conhecimento do teor de água existente no solo, podendo dessa forma controlar a quantidade de água e o momento correto de sua aplicação, conforme as necessidades da cultura (Freitas et al., 2012).

Nestas condições o manejo da irrigação deve ser realizado com o controle do teor de água no solo, fazendo o seu monitoramento com auxílio de sensores ou tensiômetros. Os sensores têm como princípio de funcionamento a capacitância elétrica e os tensiômetros levam em consideração a força de atração da água nas partículas do solo (tensão de água no solo) (Gomes et al., 2014).

A utilização de sensores e/ou tensiômetros é importante para assegurar que as plantas permaneçam constantemente em capacidade de campo, garantindo maior produtividade com consumo de água reduzido. Carvalho et al., (2012) relatam que agricultura irrigada de precisão envolve instrumentos tecnológicos (sensores). $\mathrm{O}$ uso de equipamentos para monitorar teor de água do solo em lavouras de feijão ainda é pouco difundido, embora esta cultura seja importante no Brasil e no mundo (Silva et al., 2012).

O uso de sensores com a função de monitorar umidade do solo para auxiliar no 
manejo de irrigação constituem um dos principais temas investigado na literatura (Riquelme et al., 2009, Zuojing et al., 2010, Lee et al., 2010, Cao et al., 2008, Liqiang et al., 2011). Muitos autores buscam melhorias no manejo da irrigação com finalidade de otimização uso racional da água na agricultura.

A utilização de ferramentas que auxiliem no monitoramento do teor de água no solo é importante no manejo da irrigação, pois a condição hídrica deve atender a necessidade de água da cultura. Assim sendo, quando o solo se encontra com deficiência hídrica, a água presente no sistema solo está de forma restrita acarretando danos ao metabolismo dos vegetais.

Deste modo, este trabalho teve como objetivo avaliar a funcionalidade do sensor smrt-y (Rain Bird) comparado ao tensiômetro instalados em uma cultura de feijoeiro cultivada sob deficiência hídrica e diferentes qualidades de águas em ambiente protegido.

\section{MATERIAL E MÉTODOS}

O ensaio foi conduzido no período de junho a setembro 2015, em uma estufa agrícola localizada no Instituto de BiociênciasIB/UNESP, Botucatu-SP, com coordenadas geográficas $22^{\circ} 52^{\prime}$ de latitude Sul e $48^{\circ} 26^{\prime}$ 'de longitude W Greenwich e altitude de $815 \mathrm{~m}$. O clima da cidade segundo Koppen, é temperado, quente (mesotérmico) e úmido, com inverno seco e verão chuvoso, com precipitação média anual de 1.428 mm (Cunha e Martins, 2009).

O solo utilizado foi proveniente da Fazenda de São Manuel-FCA/UNESP. A caracterização química e física do solo foi realizada mediante a análise de amostras compostas, coletadas na camada de 0 - 0,20 m de profundidade (Tabela 1 e 2). Com base nos resultados (Tabela 1), calculou-se a necessidade de calcário dolomítico para a correção do pH do solo e V\% de 70, conforme a recomendação de adubação (Aguiar et al., 2014).

Tabela 1. Análise do solo Latossolo Vermelho Amarelo coletado na Fazenda de São Manuel-SP, utilizado para o cultivo do feijoeiro

\begin{tabular}{cccccccccccccccccc}
\hline $\begin{array}{c}\mathrm{pH} \\
\mathrm{CaCl}_{2}\end{array}$ & $\begin{array}{c}\text { M.O. } \\
\mathrm{g} \mathrm{dm}^{-3}\end{array}$ & $\begin{array}{c}\mathrm{P}_{\text {resina }} \\
\mathrm{mg} \mathrm{dm}^{-3}\end{array}$ & $\mathrm{Al}^{3+}$ & $\begin{array}{c}\mathrm{H}+\mathrm{Al} \\
-\end{array}$ & $\begin{array}{l}\mathrm{K} \\
\mathrm{Cg}\end{array}$ & $\mathrm{Ca}$ & $\mathrm{Mg}$ & $\mathrm{SB}$ & $\mathrm{CTC}$ & $\mathrm{V} \%$ & $\mathrm{~S}$ & $\mathrm{~B}$ & $\mathrm{Cu}$ & $\mathrm{Fe}$ & $\mathrm{Mn}$ & $\mathrm{Zn}$ \\
\hline 5,6 & 13 & 95 & 0 & 14 & 1,8 & 14 & 5 & 21 & 35 & 59 & 17 & 0,19 & 1,3 & 60 & 4,9 & 2,2 \\
\hline
\end{tabular}

Tabela 2. Caracterização física do solo Latossolo Vermelho Amarelo coletado na Fazenda de São Manuel-SP, utilizado para o cultivo do feijoeiro

\begin{tabular}{|c|c|c|c|c|c|c|}
\hline Grossa & $\begin{array}{c}\text { Areia } \\
\text { Fina } \\
\end{array}$ & Total & Argila & Silte & Umidade & Textura \\
\hline - & - & $\mathrm{g} \mathrm{kg}^{-1}$ & ----- & ---- & $(\%)$ & \\
\hline ---- & ---- & 855 & 85 & 60 & ---- & Arenosa \\
\hline
\end{tabular}

Os equipamentos foram instalados em vasos de $25 \mathrm{~L}$, a uma profundidade de $0,15 \mathrm{~m}$ e em seguida semeou-se o feijão (cv. IAC Imperador). Os tratamentos estavam dispostos em delineamento inteiramente casualizado (DIC), constituídos de quatro tratamentos de irrigação e cinco repetições, onde: AR- água residuária (100\% da capacidade de campo do solo) monitorado com sensor e tensiômetro; C: controle com água potável $(100 \%$ da capacidade de campo do solo) monitorado com sensor e tensiômetro; DHM: Deficiência hídrica moderada com água potável (50\% da capacidade de campo do solo) monitorado com sensor e tensiômetro; DHS: deficiência hídrica severa com água potável (25\% da necessidade capacidade de campo do solo) monitorado com sensor e tensiômetro.

Para determinação da capacidade de campo (CC) utilizou-se 20 vasos. A água foi distribuída uniformemente nos vasos até que o solo ficasse saturado, em seguida coletou-se uma amostra de solo e determinou-se o peso das amostras saturadas. Vinte e quatro horas 
após saturação dos vasos, foram coletadas amostras a $20 \mathrm{~cm}$ de profundidade e pesadas. Depois levou-se as amostras para estufa por um período de 48 horas a $105{ }^{\circ} \mathrm{C}$ para determinar o peso do solo seco das amostras. Depois determinou-se a umidade do solo com base em massa.

A água residuária proveniente da estação de tratamento de esgoto da SABESP passou por um processo de filtragem e ozonização antes de ser aplicada nas plantas. A irrigação foi realizada com água potável e água de esgoto tratado via sistema de irrigação com um gotejador por vaso, com vazão de $1 \mathrm{~L} \mathrm{~h}^{-1}$. Foram utilizadas amostras deformadas que foram retiradas no local da coleta de solo e em seguida levadas ao laboratório para determinação da curva de retenção de água no solo pelo método da câmara de pressão de Richards (Figura 1). O ajuste da curva de retenção de água no solo, foi realizado pelo modelo proposto por Van Genuchten (1980). Para o monitoramento de água no solo foram instalados quatro tensiômetro na parte central do vaso e um sensor para cada tratamento. Com base nos dados coletados dos tensiômetros determinou-se quanto e quando irrigar (Gomes et al., 2015).

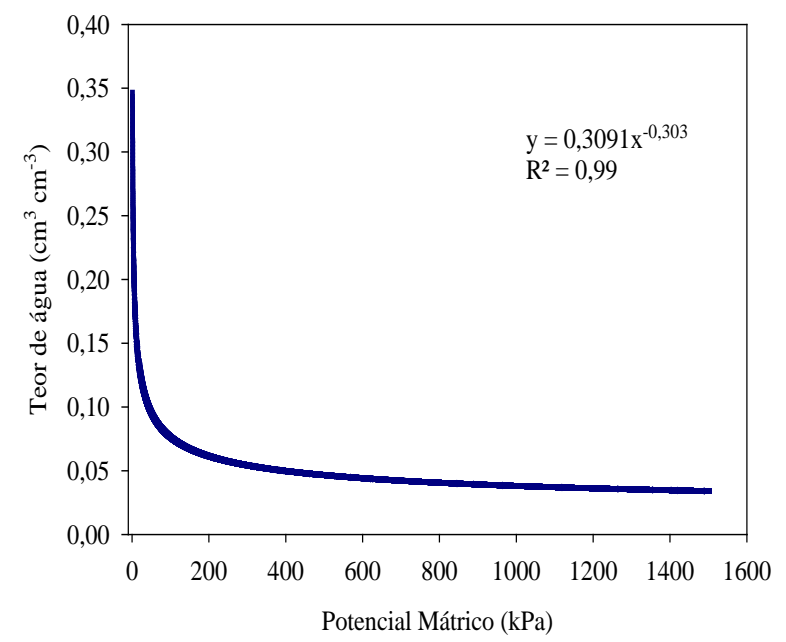

Figura 1. Curva de retenção de água do solo para o ensaio de feijão
As leituras do teor de água no solo dos sensores e dos tensiômetros foram registradas diariamente pela manhã. Para os parâmetros biométricos realizou-se duas avaliações, uma aos 35 dias após a emergência (DAE) na prefloração (antes da deficiência hídrica) e aos 80 DAE, no fim do ciclo (com deficiência hídrica).

Avaliou-se os seguintes parâmetros: teor de água no solo registrado pelo sensor (\%), teor de água no solo registrado pelo tensiômetro (\%), altura de planta $(\mathrm{cm})$, diâmetro do caule (mm), número de folhas (unidade) e área foliar. $\mathrm{Na}$ determinação deste parâmetro retirou-se todas as folhas e com auxílio de um integrador de área foliar modelo LICOR LI-3000 determinou-se área foliar total, sendo os resultados expressos em $\mathrm{m}^{2}$ planta $^{-1}$.

Os parâmetros avaliados foram submetidos à análise de variância e quando apontada significância, utilizou-se teste F. As médias foram comparadas pelo teste de Tukey a 5\% e 1\% de significância (Silva \& Azevedo, 2002).

\section{RESULTADOS E DISCUSSÃO}

Na Figura 2 o teor de água do solo expresso em \% (v/v), tanto para o tratamento com água potável (C) como para o tratamento com água residuária (AR), o manejo da irrigação do feijoeiro visava atender $100 \%$ CC, foi utilizado os dados da curva de retenção de água no solo para estabelecido os limites máximo e mínimo de armazenamento de água no solo em 19 a 16\%, no qual, baseou-se na capacidade de água disponível do solo. Observou-se que os valores de teor de água no solo (TAS) permaneceram dentro dos limites planejados para cada tratamento. Notou-se, que o tensiômetro apresentou menor oscilação quando comparado ao sensor smrt-y, nos diferentes pontos de leitura ao longo do ciclo. 

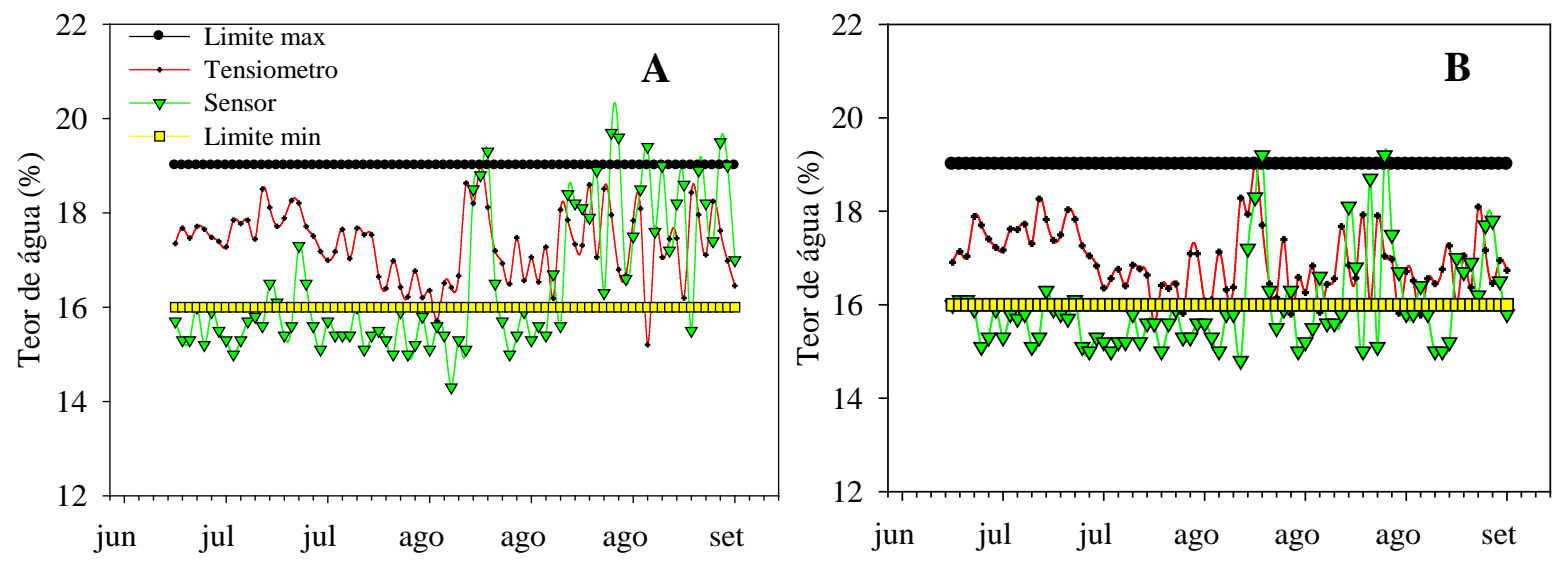

Figura 2. Teor de água no solo para os tratamentos controle (A) e água residuária (B) monitorado por sensor e tensiômetro na cultura do feijoeiro

Os valores de TAS para o tensiômetro e sensor (v/v) variaram respectivamente entre $20,2 \%$ e $14,5 \%$ para o tratamento com água potável (C) e entre $19,0 \%$ e $15,0 \%$ para o tratamento com água residuária (AR), com $100 \%$ CC. Observa-se, que em condição de boa disponibilidade hídrico, o sensor smrt-y apresentou muitos pontos com valores de TAS fora dos limites estabelecidos para o manejo da cultura. Porém, isso não causou grandes alterações na porcentagem de água no solo, pois os valores de TAS encontrados nos sensores ficaram próximos dos valores encontrados nos tensiômetros, sendo um resultado satisfatório para controle de água no solo. Freitas et al. (2012), em estudo com manejo da irrigação, utilizando sensor de umidade, obtiveram comportamento similar, em condição de alta umidade.

$\mathrm{Na}$ Figura 3A, em condições de deficiência hídrica moderada (50\% CC) foi fixado como limites máximo e mínimo de armazenamento de água no solo os valores de 11 e 9\% respectivamente. Em condições de deficiência hídrica severa (25\% CC), Figura $3 \mathrm{~B}$, estes limites fixados foram de 8,5 a $6 \%$ respectivamente. Nestas condições os valores de TAS apresentaram variação média entre $10,0 \%$ e $9,2 \%$ com DHM e entre $7,7 \%$ e $6,8 \%$ com DHS, para tensiômetro e sensor smrt-y, respectivamente.

Em condição de baixa disponibilidade hídrica tanto o sensor smrt-y quanto o tensiômetro apresentaram características de confiabilidade no quesito de monitoramento de água no solo. Segundo Persson et al. (2009), o sensor para monitoramento de água no solo ou para determinação da tensão de água no solo via TDR é de suma importância no manejo de irrigação. Gomes et al. (2013) observaram que independentemente do método empregado (tensiometria ou sensor de umidade) o monitoramento da umidade do solo para os diferentes métodos apresentaram correlação de 0,72 , assim, constatou-se que ambos os métodos podem ser utilizados.

Para as variáveis biométricas do feijoeiro (Tabela 3), o diâmetro de caule (DC) não diferiu nos diferentes tratamentos hídricos aos 35 DAE e 80 DAE. Os valores de DC variaram entre 4,45 a 5,76 mm. Vale et al. (2012) avaliando a tolerância ao estresse hídrico em feijão observaram que DC não diferiu estatisticamente em função do estresse hídrico. Junior et al. (2014) e Cunha et al. (2015) constaram que o diâmetro de caule é menor para as plantas sob deficiência hídrica.

O número de folhas por planta (NFP) e área foliar da planta (AF) apresentaram diferença $(\mathrm{P}<0,01)$ para 80 DAE. Nota-se que as plantas do tratamento AR apresentou maior NFP e AF. Silva et al. (2009), estudando a densidade de plantas observaram que número de folhas apresenta um crescimento linear.

Os menores valores AF e NFP apresentados pelo tratamento DHS, mostra que a restrição hídrica promoveu danos a cultura de forma a reduzir a área fotossintética da planta em virtude da necessidade de reduziras perdas de água para o ambiente. Bonfim et al. (2012) 
constataram que o estresse hídrico em feijoeiro promove a redução da área foliar e do número de folhas, de acordo com a intensidade do estresse hídrico recebido pela planta. Oliveira et al. (2013) avaliaram a interação entre bioestimulante e a salinidade em feijoeiro e obtiveram valores médios de $0,188 \mathrm{~m}^{2}$ planta $^{-}$ 1 .

A altura da planta (AP) apresentou diferença significativamente $(\mathrm{P}<0,01)$ para os tratamentos hídricos AR, C e DHS aos 80
DAE. Logo, os diferentes teores de água no solo influenciaram na AP de forma a alterar sua biômetra. Sousa et al. (2010) observaram a influência da supressão da irrigação em estádios de desenvolvimento do feijoeiro e constatou que a deficiência hídrica no estádio vegetativo interfere na altura das plantas. Gonçalves et al. (2015) e Gava et al. (2015), observaram que o feijoeiro submetido a deficiência hídrica apresenta menor altura em relação as plantas em capacidade de campo.

Tabela 3. Análise biométrica do feijoeiro, diâmetro do caule (DC), número de folhas por plantas (NFP), área foliar da planta (AF), altura de planta (AP) em função dos tratamentos hídricos para cada época de avaliação

\begin{tabular}{|c|c|c|c|c|c|c|c|c|c|c|c|c|c|c|}
\hline \multirow[t]{2}{*}{ TRAT. } & \multicolumn{3}{|c|}{$\begin{array}{c}\text { DC } \\
\left(\mathrm{mm} \mathrm{planta}^{-1}\right)\end{array}$} & \multicolumn{4}{|c|}{$\begin{array}{c}\text { NFP } \\
\text { (unidade) }\end{array}$} & \multicolumn{4}{|c|}{$\begin{array}{c}\text { AF } \\
\left(\mathrm{m}^{2} \text { planta }^{-1}\right)\end{array}$} & \multicolumn{3}{|c|}{$\begin{array}{c}\text { AP } \\
\left(\text { cm planta }^{-1}\right)\end{array}$} \\
\hline & $35 \mathrm{DAE}$ & $80 \mathrm{D}$ & & $35 \mathrm{I}$ & $\mathrm{AE}$ & $80 \mathrm{I}$ & $\mathrm{AE}$ & $35 \mathrm{DA}$ & & $80 \mathrm{DA}$ & & $35 \mathrm{D}$ & & $80 \mathrm{DAE}$ \\
\hline AR & $4,63 \quad \mathrm{a}$ & 5,63 & $\mathrm{a}$ & 18 & $\mathrm{a}$ & 52 & $\mathrm{a}$ & 0,092 & $\mathrm{a}$ & 0,208 & $\mathrm{a}$ & 43,6 & $\mathrm{a}$ & $62,2 \quad \mathrm{a}$ \\
\hline $\mathrm{C}$ & 4,55 & 5,52 & $\mathrm{a}$ & 15 & $\mathrm{a}$ & 35 & b & 0,085 & $\mathrm{a}$ & 0,130 & b & 40,7 & $d$ & 52,2 \\
\hline DHM & 4,48 & 4,79 & $\mathrm{a}$ & 19 & $\mathrm{a}$ & 33 & b & 0,095 & $\mathrm{a}$ & 0,101 & b & 41,0 & $\mathrm{a}$ & 48,5 bc \\
\hline DHS & 4,70 & 4,45 & a & 16 & $\mathrm{a}$ & 19 & C & 0,089 & $\mathrm{a}$ & 0,029 & C & 40,7 & $\mathrm{a}$ & $43,8 \quad$ c \\
\hline
\end{tabular}

As médias na vertical com letra minúsculas na coluna é a comparação dos tratamentos hídricos pelo teste Tukey a 5\%; DAE: dias após emergência; AR: irrigação com água residuária, C: irrigação com água potável, DHM: deficiência hídrica moderada com água potável; DHS: deficiência hídrica severa com água potável.

\section{CONCLUSÃO}

O sensor smrt-y juntamente com o tensiômetro apresentaram viabilidade no monitoramento e controle do teor de água do solo, auxiliando no manejo de irrigação independentemente do tipo de água utilizada.

A deficiência hídrica provocou danos nas plantas de feijoeiro de forma a modificar suas características biométricas.

O sensor remoto apresentou confiabilidade para as medidas, comparável ao método de aferição por tensiômetro.

\section{REFERÊNCIAS BIBLIOGRÁFICAS}

AGUIAR, A. T. E.; GONÇALVES, C.; AYRES, M. E.; PATERNIANI, G. Z. Instruções agrícolas para as principais culturas econômicas. $7^{\mathrm{a}} \mathrm{Ed}$. Campinas: Instituto Agronômico (Boletim IAC, $\mathrm{n}^{\circ}$ 200), 2014. 452 p.
AZEVEDO, C. V. G.; RIBEIRO, T.; SILVA, D. A.; CARBONELL, S. A. M; CHIORATO, A. F. Adaptabilidade, estabilidade e resistência a patógenos em genótipos de feijoeiro. Pesq. agropec. bras., Brasília, v. 50, n. 10, p. 912922, out. 2015.

BONFIM, F. P. G.; CASALI, V. W. D.; MENDONÇA, E. G.; MARTINS, R. E. Estresse hídrico em feijoeiro (Phaseolus vulgares L.) tratado com o preparados homoepáticos de Arnica montana. Revista Enciclopédia Biosfera, v. 14, n. 8, p. 530-538, 2012.

CAO, X.; CHEN, J.; ZHANG Y.; SUN, Y. Development of an integrated wireless sensor network micro-environmental monitoring system. ISA Transactions, v. 47, n. 3, p. 247255, 2008.

CARVALHO, F. B. S.; LEAL, B. G.; SANTOS FILHO, J. V.; BAIOCCHI, O. R.; LOPES, W. T. A.; ALENCAR, M. S. 
Aplicações Ambientais de Redes de Sensores Sem Fio. Revista de Tecnologia da Informação e Comunicação, n. 2, p. 14-19, 2012.

CUNHA, A. R.; MARTINS, D. Classificação climática para os municípios de Botucatu e São Manuel, SP. Irriga, v. 4, n. 1, p. 1-11, 2009.

CUNHA, R. P.; CORRÊA, M. F.; SCHUCH, L. O. B.; OLIVEIRA, R. C.; JUNIOR, J. S. A.; SILVA, J. D. G.; ALMEIDA, T. L. Diferentes tratamentos de sementes sobre o desenvolvimento de plantas de soja. Ciência Rural, v. 45, n. 10, p. 1761-1767, 2015.

FREITAS, W. A.; CARVALHO, J. A.; BRAGA, R. A.; ANDRADE, M. J. B. Manejo da irrigação utilizando sensor da umidade do solo alternativo. Revista Brasileira de Engenharia Agrícola e Ambiental, v. 16, n. 3, p. 268-274, 2012.

GAVA, R.; FRIZZONE, J. A.; SNYDER, R. L.; JOSE, V. J.; JUNIOR, E. F. F.; PERBONI, A. Estresse hídrico em diferentes fases da cultura da soja. Revista Brasileira de Agricultura Irrigada, v. 9, n. 6, p. 349-359, 2015.

GOMES, E. P.; OLIVEIRA, G. Q.; SCHWERZ, F.; SILVA, P. A.; BISCARO, G. A.; SANTOS, R. C. Avaliação do medidor eletrônico de umidade do solo "hidrofarm" em latossolo vermelho distroférrico. Revista Brasileira de Agricultura Irrigada, v. 7, n. 1, p. 85-90, 2013.

GOMES, E. R.; BROETTO, F.; QUELUZ, J.G.T.; BRESSAN, D. F. Efeito da fertirrigação com potássio sobre o solo e produtividade do morangueiro, Irriga, Edição Especial, 20 anos Irriga + 50 anos FCA, p. 107122, 2015.

GOMES, E. R.; SILVA, L. R. A.; ANDRADE JÚNIOR, A. S.; SOUSA, V. F.; MOUSINHO, F. E. P. Potencial hídrico foliar da melancieira em resposta a variação no conteúdo de água no solo. Irriga, n. 1, p. 29-39, 2014.
GONÇALVES, J. G. R.; CHIORATO, A. F.; SILVA, D. A.; ESTEVES, J. A. F.; BOSETTI, F.; CARBONELL, S. A. M. Análise da capacidade combinatória em feijoeiro comum submetido ao déficit hídrico. Bragantia, v. 74, n. 2, p. 149-155, 2015.

JUNIOR, A. A. B.; PROCÓPIO, S. O.; DEBIASI, H.; FRANCHINI, J. C. Redução do espaçamento entre linhas na cultura da soja. Londrina, PR: Embrapa Soja, (Circular Técnica, 106), 2014. 8 p.

LEE, W. S.; ALCHANATIS, V.; YANG, C.; HIRAFUJI, M.; MOSHOU, D.; LI, C. Sensing technologies for precision specialty crop production. Computers and Electronics in Agriculture, v. 74, p. 2-33, 2010.

LIQIANG, Z.; SHOUYI, Y.; LEIBO, L.; ZHEN, Z.; SHAOJUN, W. A Crop Monitoring System Based on Wireless Sensor Network. Procedia Environmental Sciences, v. 11, p. 558-565, 2011.

OLIVEIRA, F. A.; MEDEIROS, J. F.; OLIVEIRA, M. K. T.; SOUZA, A. A. T.; FERREIRA, J. A.; SOUZA, M. S. Interação entre salinidade e bioestimulante na cultura do feijão caupi. Revista Brasileira de Engenharia Agrícola e Ambiental, v. 17, n. 5, p. 465-471, 2013.

PERSSON, M.; WRAITH, J. M.; DAHLIN, T. A. Small-scale matricpotential sensor based on time domain reflectometry. Soil Science Society of America Journal, v. 70, p. 533536, 2006.

RIQUELME, J. A. L.; SOTO, F.; SUARDÍAZA, J.; SÁNCHEZA, P.; IBORRA, A.; VERA, J. A. Wireless Sensor Networks for precision horticulture in Southern Spain. Computers and Electronics in Agriculture, v. 68, n. 1, p. 25-35, 2009.

SILVA, A. F.; CONCENÇO, G.; ASPIAZÚ, I.; FERREIRA, E. A.; GALON, L.; COELHO, A. T. C. P.; SILVA, A. A.; FERREIRA, F. A. Interferência de plantas daninhas em diferentes 
densidades no crescimento da soja. Planta Daninha, v. 27, n. 1, p. 75-84, 2009.

SILVA, F. A. S. E.; AZEVEDO, C. A. V. Versão do programa computacional Assistat para o sistema operacional Windows. Revista Brasileira de Produtos Agroindustriais, v. 4, n. 1, p. 71-78, 2002.

SILVA, J. C.; ARF, O.; GERLACH, G. A. X.; KURYIAMA, C. S.; RODRIGUES, R. A. F. Efeito hormese de glyphosate em feijoeiro. Pesquias Agropecuária Tropical, v. 42, n 3, p. 295-302, 2012.

SOUSA, M. A.; LIMA, M. D. B. Influência da supressão da irrigação em estádios de desenvolvimento do feijoeiro cv. carioca comum. Bioscience Journal, v. 26, n. 4, p. 550-557, 2010.
VALE, N. M.; BARILI, L. D.; ROZZETO, D. S.; STINGHIN, J. C.; COIMBRA, J. L. M.; GUIDOLIN, A. F.; KÖOP, M. M. Avaliação para tolerância ao estresse hídrico em feijão. Revista Biotemas, v. 25, n. 3, p. 135-144, 2012.

VAN GENUTCHEN, M. T. H. A closed-from equation for predicting the hydraulic conductivity of insatured. Soil Science Society American Journal, v. 41, p. 892-898, 1980.

ZUOJING, Z.; HAIHUI, Z. Design of wireless monitoring and warning system for protected agriculture environment. In: 6th International Conference on Wireless Communications Networking and Mobile Computing, Chengdu City, Anais...Chengdu City, China, 2010. p.1-5. 